\title{
Rapid Manufacturable Ventilator for Respiratory Emergencies of COVID-19 Disease
}

\author{
J. Tharion ${ }^{1} \cdot$ S. Kapil ${ }^{2} \cdot$ N. Muthu² ${ }^{2}$ J. G. Tharion ${ }^{3} \cdot$ S. Kanagaraj ${ }^{2}$
}

Received: 17 April 2020 / Revised: 27 May 2020 / Accepted: 29 May 2020 / Published online: 7 June 2020

(C) Indian National Academy of Engineering 2020

\begin{abstract}
Influenza like pandemics are a severe threat to any established health care system as many thousands of patients would need emergency ventilator support during the acute respiratory failure stage, and this quickly overloads the existing facilities. The present article addresses the design and development of a human breathing assist machine (ventilator) prototype for use by qualified medical professionals in the emergency room, as well as in other locations, where a regular ventilator machine cannot be made available. The ventilator has been designed using readily available locally sourced materials, which can be assembled in a short time. This ensures the minimum required features to ventilate a patient in emergency conditions. The popular crank-rocker mechanism has been used to meet some of the vital design requirements of the emergency ventilator. The size of the links has been chosen to maintain a fixed inspiratory-to-expiratory (I:E) time ratio of 1:2. The kinematic linkage design has been kept modular by introducing a feature to adjust the location of the rocker tip to control the tidal volume from $100 \mathrm{ml}$ to $600 \mathrm{ml}$ of oxygenated air per breath. A virtual CAD model, based on the above-mentioned linkage design, has been designed to assess the variation of the position and velocity with time. Finally, a working prototype has been made, and it was observed that the I:E time ratio of 1:2 was achieved satisfactorily.
\end{abstract}

Keywords COVID-19 $\cdot$ Ventilator $\cdot$ Piston $\cdot$ Crank rocker $\cdot$ Low cost

\section{Introduction}

COVID-19 pandemic has the potential for a rapid outburst where emergency medical care has to be re-organised so as to meet the spiked respiratory support needed for the critically ill. In such a scenario, the ventilator must be rapidly manufacturable and deployable. Even otherwise, the need for ventilators is accelerated due to air pollution-related lung diseases that are on the rise in the developing countries (Ait-Khaled et al. 2001). Mechanical ventilators are the machines that assist the patients with severe acute respiratory distress; they enable an artificial exchange of oxygen and carbon dioxide in the lungs (WebMD 2008). While there

N. Muthu

nelsonm@iitg.ac.in

1 NECBH Section, Indian Institute of Technology Guwahati, Guwahati, Assam, India

2 Department of Mechanical Engineering, Indian Institute of Technology Guwahati, Guwahati, Assam, India

3 Department of Anaesthesia, V.M.M.C. and Safdarjung Hospital, New Delhi, India are sophisticated ventilators with advanced features that cost up to INR 20 lakhs, the aim of this paper is to describe a quick construction of a low-cost ventilator to ventilate patients on oxygenated air mixture through an endotracheal tube, for the most severe hypoxemic phase of the COVID-19 disease, also known as severe acute respiratory syndrome (SARS) or acute respiratory distress syndrome (ARDS). In COVID-19 disease, approximately $5 \%$ of the infected people are expected to get ARDS (Cao 2020).

There are many designs of the emergency and portable ventilators in the market, which are one order cheaper than the hospital ventilators (Husseini et al. 2010). They are broadly classified into two types: pneumatic and electric. The pneumatic ventilators require externally pressurized air, which may not be readily available in emergencies. On the other hand, the modern electrically operated ventilators can operate anywhere. However, due to its intricate design and function, they are generally costlier than the pneumatic ones.

In this article, the minimalistic design specifications for a clinically acceptable ventilator have been obtained from Medicines and Healthcare products Regulatory agency (MHRA), UK specifically for the "Rapidly Manufactured 
Ventilator System (RMVS)" intended for use in UK hospitals during the current COVID-19 pandemic caused by SARS-CoV-2 virus [https://assets.publishing.service.gov. uk/government/uploads/system/uploads/attachment_data/ file/876167/RMVS001_v3.1.pdf (as on 15-04-2020)], which was released as early as March 18th, 2020. Also, the DRDO guidelines were available on a later date [https://www. mohfw.gov.in/pdf/EssentialTechfeaturesforVentilators.pdf (as on 18-05-2020)]. There are three recommended modes of continuous mode ventilation: pressure regulated volume control (PRVC), pressure controlled ventilation (PCV), and volume controlled ventilation (VCV). The VCV being the most basic of the ventilator modes, is used in the present work. In the VCV, the user sets a tidal volume and controls the pressure limits.

The recommended tidal volumes for ARDS are 4-6 ml/ $\mathrm{kg}$ body weight during one inspiratory cycle (Retamal et al. 2013; Network et al. 2000), which for a $30 \mathrm{~kg}$ Indian woman could be as low as 120-180 ml. This will be even lower for the adolescent age group with lower body weight. Therefore, in the present work, the lower limit of tidal volume is kept as $100 \mathrm{ml}$. The maximum tidal volume is set at $600 \mathrm{ml}$ as per the DRDO guidelines.

A mechanical design for flow control, rate control, and other controls are worked upon here, over an electronic sensor-based model, as the complexity in design and difficulty in the procurement of components can hinder deployment of sufficient ventilators during an epidemic (Darwood et al. 2019). It has to be mentioned that many designs for rapidly prototyped ventilators being developed currently for use in this epidemic are based on the modification of an AMBU (Artificial Manual Breathing Unit) bag, with an automated compression mechanism. On initial consideration, this appears to be a viable option, but considering that precise volume control of ventilator output is a critical requirement when ventilating an intubated patient, the AMBU bag design poses problems. Due to the physical shape variations (due to the design variations among brands) in the available AMBU bags, adjustments would have to be made to the mechanical system to accommodate the differences in them. Hence, in our opinion, the use of a standardized air reservoir (in the form of a cylinder) can streamline end-user training, improving usability and compliance, which can be critical for use in emergencies.

The most commonly used mechanical anaesthesia ventilators are either bellow-driven or piston-driven. Some of the classical bellow-driven ventilators are reported in (Tobia 1997; Hedenberg 2011). The classical pistondriven ventilators are reported in (Power 1997; Power et al. 1996). Both the type of ventilators is found to be in good agreement in delivering a low amount of tidal volume (Bachiller et al. 2008). However, it has been observed that the piston-driven ventilators are quiet and accurate (in tidal volume delivery) as compared to the bellow-driven ventilators (Jain and Swaminathan 2013). In this work, a cylinder-diaphragm unit is used.

To maintain an inspiratory-to-expiratory time ratio of 1:2 and a variable tidal volume, a six-bar planar linkage based on crank-rocker mechanism has been used. This mechanism can be observed in several real-life machines such as rock-crusher mechanism, packing mechanism, oscillating sanding drum, door closing mechanism, windshield-wiping mechanism, etc. The main thrust of this article is the synthesis of this six-bar mechanism and the assembling of the cylinder diaphragm with the linkage for obtaining the desired output. In the passing, some comments on the additional pressure limiting requirements and the ways to achieve it are also made.

\section{Device Design}

This design assumes the availability of medical grade oxygen supply (100\%) at pressures equivalent to that available at standard hospital Intensive Care Unit Settings ( $~ 50 \mathrm{psi})$. There are three modules in this design: a driving mechanism that governs the respiratory rate, the kinematic linkage that controls the tidal volume and the I:E ratio, and the cylinder-diaphragm unit that supplies oxygenated air mixture at the desired fraction of inspired oxygen $\left(\mathrm{FiO}_{2}\right)$ value.

\section{Driving Mechanism}

The primary driving mechanism can be a simple electric motor (simple geared DC or stepper motor). The motor will be able to rotate at $10-30 \mathrm{rpm}$. A single rotation of the motor corresponds to a single breath cycle.

In the present design, inspiratory and expiratory time are different and is a vital feature. Achieving a different time for inspiration and expiration using a pneumatic drive increases the complexity of the pneumatic circuit, as well as increases the cost. Hence, a motor-based design had to be finalized.

One of the objectives of the article is to minimize the usage of electronic components. Therefore, electronic speed control has not been used here. Instead, a geared DC motor with a wide working voltage range, sufficient torque, and a high base rpm along with a high reduction ratio is used. This enables us to achieve a final rpm of up to 30 . This strategy offers a substantial mechanical advantage, and the resultant torque magnification achieved through the gears will act as a buffer, resulting in the output rpm stability of the motor, despite changes in the pressure load. 


\section{Kinematic Linkage}

The primary ventilatory targets in the ARDS stage include the attainment of the gas exchange value of $\mathrm{PaCO}_{2}$ of $<80 \mathrm{mmHg}$, and $\mathrm{PaO}_{2}$ of $55-80 \mathrm{mmHg}$ (Hess and Kacmarek 2014). The rationale of using mechanical ventilation here is to achieve the ventilatory targets while ensuring a minimal lung injury in the process, which requires a finer volume control, over and above the initial set volume. Hence, to achieve continuously adjustable volume control in a mechanical device, a six-bar linkage mechanism has been adopted. Furthermore, for an emergency ventilator, it is attempted to achieve these functionalities using a limited choice of materials which can be locally machined or sourced to reduce the assembly time. According to this design paradigm, only the very critical ventilator features necessary for managing the acute stage of COVID-19 are incorporated, leaving many special ventilator features, so as to be driven based on a design comprising maximally of mechanical components.

Generally, a variable I:E time ratio ranging from 1:1 to 1:5 is preferred in the ventilators to treat a broad spectrum of respiratory diseases, including COVID-19 [https://www. medscape.com/answers/810126-45486/what-is-the-norma 1-inspiration-and-expiration-ratio-in-mechanical-ventilatio $\mathrm{n}$ (as on 18-05-2020)]. For example, an I:E ratio of 1:4 is needed in the case of Asthma/COPD. In the present work, the I:E time ratio is kept as 1:2, i.e., expiration lasts twice as long as inspiration. Second, the ventilator should be capable of delivering a tidal volume between $100 \mathrm{ml}$ and $600 \mathrm{ml}$ of oxygenated air per breath.

An offset slider-crank mechanism may seem to be the best choice to achieve the above-mentioned specifications.
However, if the ventilator has to cater to a broad spectrum of respiratory conditions, the slider crack fails to provide time ratio equal to or lesser than 1:3. Due to the design requirements of the ventilator with a possibility to expand it later to treat more than one disease, a six-bar mechanism has been considered here. Furthermore, the primary need for COVID-19 pandemic ventilator is to have an I:E time ratio of $1: 2$ with a variable volume, which is difficult to realize using a slider-crank mechanism. To meet the specifications, a six-bar kinematic linkage has been proposed, as shown in Fig. 1. The six-bar linkage has been synthesized in the following three stages:

1. The four-bar linkage (ABCE) was designed using a graphical method. It is a classical rocker-amplitude problem with the desired I:E time ratio (Waldron et al. 2016).

2. The fixed pivot of the four-bar linkage is then converted to a moving pivot in such a way that all the link lengths remains constant.

3. The fixed pivot $\mathrm{D}$ of a slider-crank mechanism (DFG) was then appropriately selected on the output link CE, such that the piston motion can provide desirable tidal volume.

The degrees of freedom (DOF) of the planar linkage can be calculated by Kutzbach equation $F=3(n-1)-2\left(P_{1}\right)-P_{2}$, where $F$ is the degrees of freedom, $n$ is the number links, $P_{1}$ is the number of 1 DOF joints, and $P_{2}$ is the number of 2 DOF joints. In the planar linkage shown in Fig. $1, n=6, P_{1}=6$, and $P_{2}=1$, and hence, the DOF of this mechanism will be $F=2$. It
Fig. 1 Kinematic linkage of the proposed ventilator

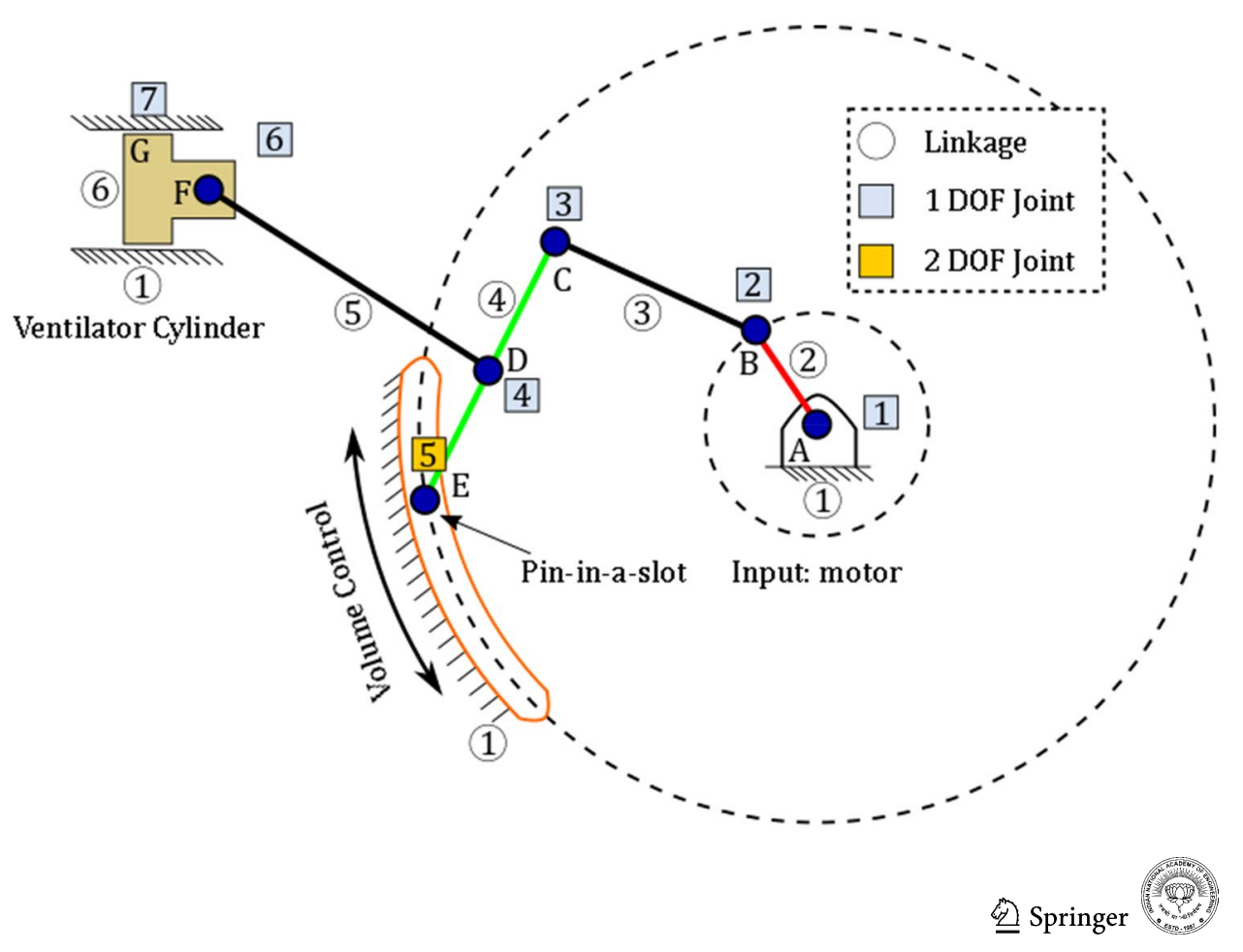


indicates that there should be two inputs (one from the motor at point ' $A$ ' and another at point ' $E$ ') to the system to assemble the linkage uniquely. The current design incorporates an offset slider which may increase the load on the motor. Steps will be taken to use an in-line slider in a later, advanced version.

Figure 2a shows the position with time plot of the link 6 at $100 \mathrm{rpm}$ obtained by simulation. It is evident that the compression and expansion times are in the ratio of 1:2. The tidal volume can be increased by increasing the stroke length (Fig. 2b). It should be emphasized that the I:E ratio of 1:2 can also be obtained by controlling the driving motor. However, programming it and setting up the user interface control platform involves time and expertise. Since the objective of the present work is to construct a basic ventilator quickly with less expertise, the proposed linkage would be handy.

\section{Cylinder-Diaphragm Unit}

A cylinder-diaphragm unit is connected to the six-bar planar linkage. For a better visualization, in some hospital ventilators, this cylinder is made of a transparent material in which the diaphragm movement can be seen in real time. In the present prototype, the cylinder is made of an acrylic sheet of $170 \mathrm{~mm}$ diameter with flanges on either end. The flanges help in fixing the diaphragm using an acrylic ring and a clamp-type mechanism. The diameter of the cylinder is selected such that the stroke length of the piston can be kept under the material tolerance limits of the diaphragm, based on the tidal volume requirement.

In addition to the above-mentioned three modules, the ventilator must have pressure limiting options. The ventilator requires precise control on pressure limits comprising of regulation of the inspiratory cut-off pressures (15-40 cm water) and a variable positive end expiratory pressure (PEEP) $(5-20 \mathrm{~cm}$ water). A straightforward way of achieving this is using an adjustable water manometer-based valve, which can be quickly developed in the lab settings. This will increase the dimensions of the system. The other possible solutions would be to make use of a low stiffness spring/weight-based valve. However, in the latter, the calibration may be timeconsuming. For safety reasons, an alarm to alert is required (1) when the inspiratory pressure cut-off is reached, (2) machine switched off while in continuous mandatory ventilation (CMV) mode. Also, the ventilator must have a battery backup of $20 \mathrm{~min}$.

Without taking into consideration the time for procuring the components, the approximate time for assembling and testing the ventilator will be from 2 to 4 weeks. The cost would approximately be Rs. 40,000.

The schematic of the entire ventilator system is shown in Fig. 3. Some important valves are shown with its description. A preliminary prototype consisting of the links and the cylinder (Fig. 4a, b) has been made using acrylic material. The diaphragm is made of silicone rubber in the lab settings, and this is attached to the cylinder in between the flanges using bolts. The transit positions of the manufactured linkage were observed, as shown in Fig. 5a, b, and the Time Ratio $(T R)$ can be calculated as:

$\mathrm{TR}=\frac{360^{\circ}-235^{\circ}}{235^{\circ}}=\frac{125^{\circ}}{235^{\circ}}=\frac{25^{\circ}}{47^{\circ}} \approx 0.53$.

The angular displacements were measured using a protractor. However, linkages were designed for a TR of 1:2. A difference in the time ratio of 0.03 is attributed to manufacturing error in linkages that were made using a portable jigsaw machine.

There can be multiple obstacles in the deployment of an emergency ventilator. Some of the important hurdles are: (1) training medical professionals and nursing staff for using the ventilator. The functionality and form factor of
Fig. 2 a 1:2 I:E ratio of the link 6 at $100 \mathrm{rpm}$. b Tidal volume control at 1:2 (I:E) ratio

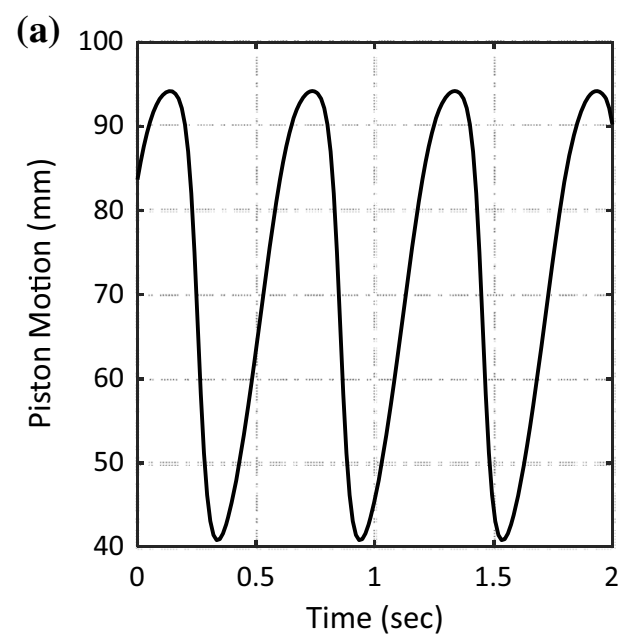


Fig. 3 Schematic of the mechanical ventilator system

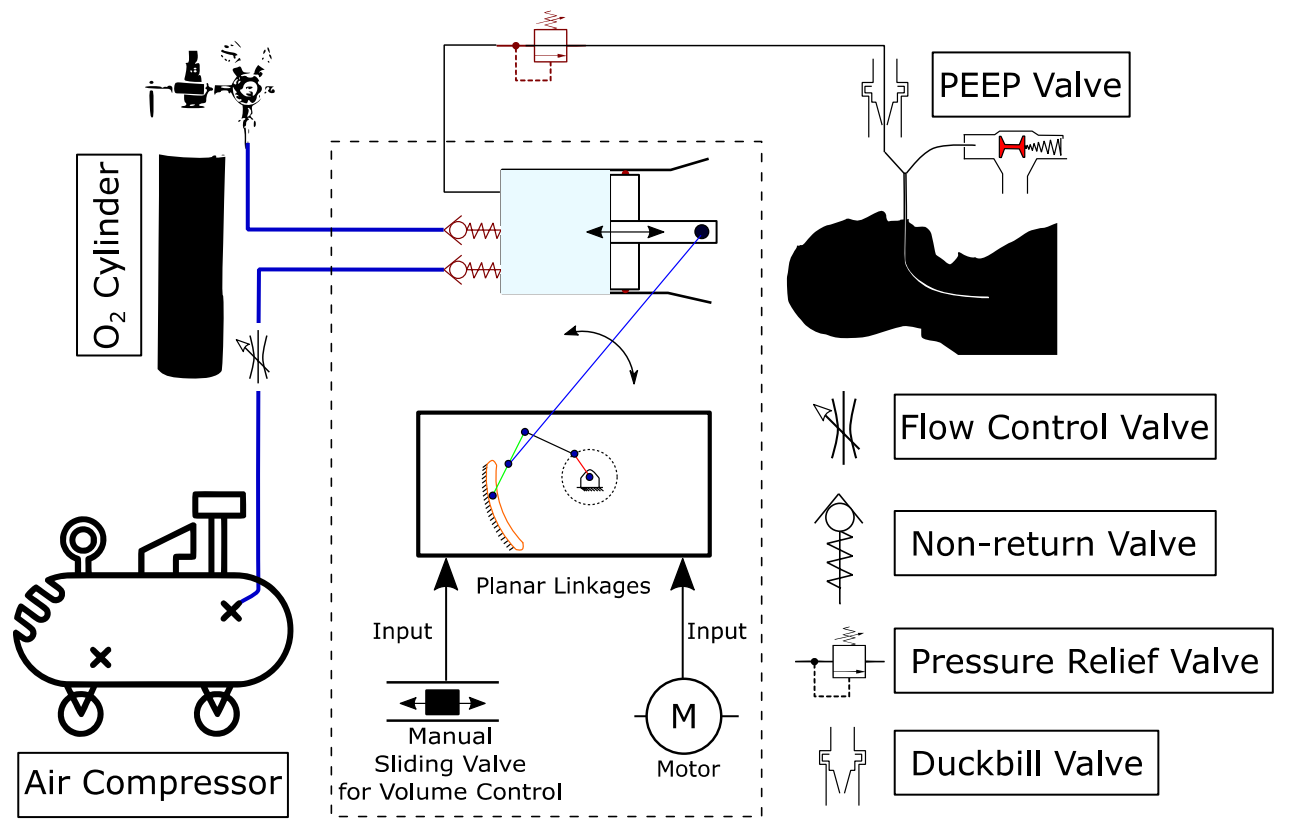

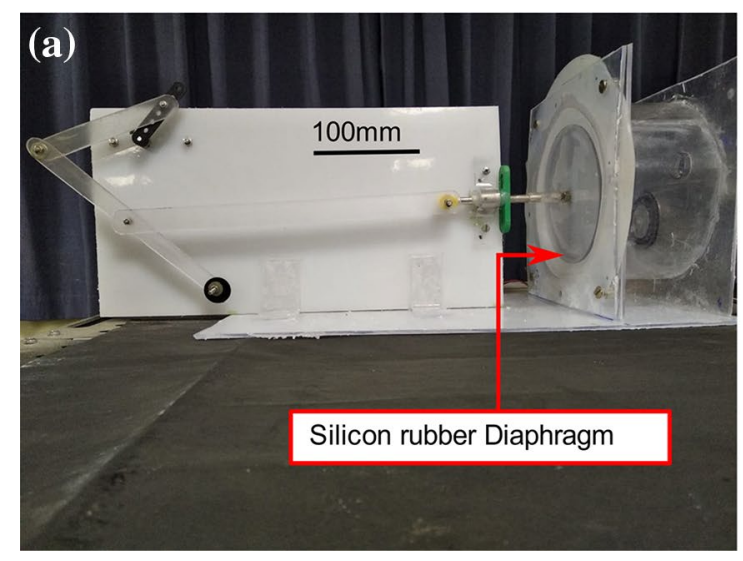

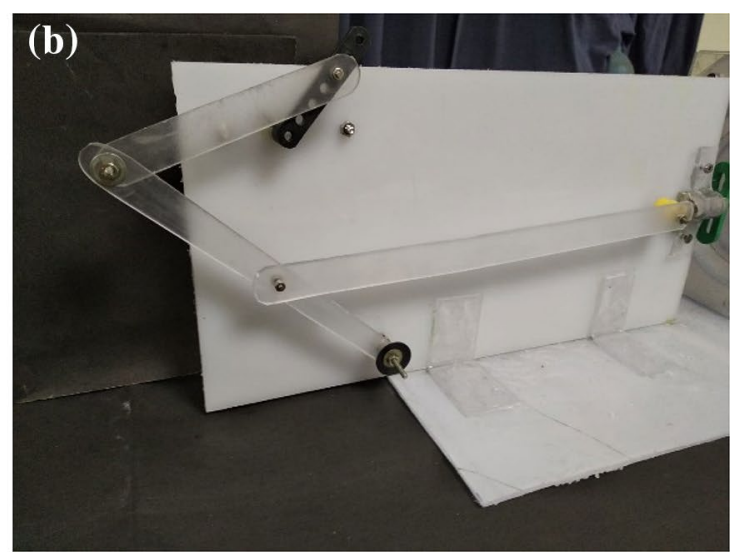

Fig. 4 a Preliminary prototype of the ventilator system. b Planar linkage
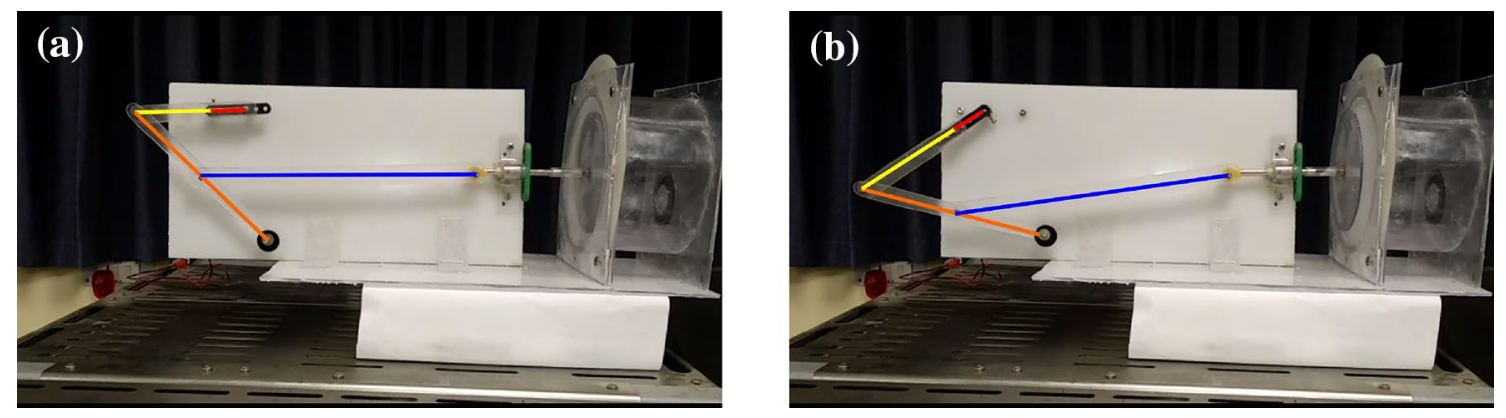

Fig. 5 Ventilator at two extreme positions a Forward stroke. b Backward stroke

the emergency ventilator will be different from the standard high-end ventilators. Therefore, in case of an emergency deployment of ventilators, training time may be insufficient.
(2) Ventilator weaning - a procedure necessary before the patient is taken out of prolonged ventilator therapy, is not incorporated in the current model. This requires unique 
advanced modes. Hence, difficulty would be encountered while removing the patient from long-term ventilation.

\section{Conclusion}

A quickly constructible ventilator design with user-controlled tidal volume at 1:2 (I:E ratio) has been proposed. This has been achieved by a six-bar planar linkage. The tip of the rocker can be relocated along the circular groove to adjust the tidal volume. A prototype has been made that demonstrates the simplicity and reliability of the approach to driving the emergency mechanical ventilators in low resource environments. Furthermore, this pragmatic design leads to cost-effectiveness. It is envisaged that this technology may provide access to simple ventilation in healthcare settings that are unable to procure advanced ventilators in emergency scenarios. A difference of 0.03 in time ratio has been observed in the prototype. Metallic links of the desired length, manufactured in the workshop, will be used instead of flexible acrylic links to reduce any error in the time ratio, in a later version. Attempts are currently made to incorporate an in-line slider and variable time ratio. It must be noted that the current design is not intended for permanent use, but as an emergency measure to overcome the expected acute shortage of ventilators as a result of the currently existing worldwide outbreak of SARS-CoV2.

Acknowledgements This work is supported by the North East Centre for Biological Sciences and Healthcare Engineering (NECBH), Department of Biotechnology, Government of India.

\section{References}

https://assets.publishing.service.gov.uk/government/uploads/syste m/uploads/attachment_data/file/876167/RMVS001_v3.1.pdf. Accessed 15 Apr 2020

https://www.medscape.com/answers/810126-45486/what-is-the-norma 1-inspiration-and-expiration-ratio-in-mechanical-ventilation. Accessed 18 May 2020

https://www.mohfw.gov.in/pdf/EssentialTechfeaturesforVentilators.pdf. Accessed 18 May 2020
Aït-Khaled N, Enarson D, Bousquet J (2001) Chronic respiratory diseases in developing countries: the burden and strategies for prevention and management. Bull World Health Organ 79(10):971-979

Al Husseini AM, Lee HJ, Negrete J, Powelson S, Servi A, Slocum A, Saukkonen J (2010) Design and prototyping of a low-cost portable mechanical ventilator. In: Proceedings of the 2010 design of medical devices conference, DMD2010 April 13-15, Minneapolis, MN, USA. https://web.mit.edu/2.75/projects/DMD_2010_ Al_Husseini.pdf

Bachiller PR, McDonough JM, Feldman JM (2008) Do new anesthesia ventilators deliver small tidal volumes accurately during volumecontrolled ventilation? Anesth Analg 106(5):1392-1400

Cao X (2020) COVID-19: immunopathology and its implications for therapy. Nat Rev 20:269-270

Darwood A, McCanny J, Kwasnicki R, Martin B, Jones P (2019) The design and evaluation of a novel low-cost portable ventilator. Anaesthesia 74:1406-1415

Hedenberg H (2001) U.S. Patent No. 6,263,873. U.S. Patent and Trademark Office, Washington, DC

Hess DR, Kacmarek RM (2014) Essentials of mechanical ventilation, 3rd edn. McGraw-Hill Education/Medical, USA

Jain RK, Swaminathan S (2013) Anaesthesia ventilators. Indian J Anaesth 57(5):525

Network ARDS, Brower RG, Matthay MA et al (2000) Ventilation with lower tidal volumes as compared with traditional tidal volumes for acute lung injury and the acute respiratory distress syndrome. N Engl J Med 342(18):1301-1308

Power JS (1997) U.S. Patent No. 5,673,689. U.S. Patent and Trademark Office, Washington, DC

Power JS, O'mahony JJ, Merrick EB (1996) U.S. Patent No. 5,531,221. U.S. Patent and Trademark Office, Washington, DC

Retamal J, Libuy J, Jiménez M et al (2013) Preliminary study of ventilation with $4 \mathrm{ml} / \mathrm{kg}$ tidal volume in acute respiratory distress syndrome: feasibility and effects on cyclic recruitment-derecruitment and hyperinflation. Crit Care 17(1):R16

Tobia RL (1997) U.S. Patent No. 5,651,360. U.S. Patent and Trademark Office, Washington, DC

Waldron KJ, Kinzel GL, Agrawal SK (2016) Kinematics, dynamics, and design of machinery hardcover, 3rd edn. John Wiley \& Sons Inc, United States

WebMD, coedited by William CS, Melissa CS (2008) Webster's new world $^{\mathrm{TM}}$ medical dictionary, 3rd edn. Wiley Publishing, Inc., Hoboken, New Jersey

Publisher's Note Springer Nature remains neutral with regard to jurisdictional claims in published maps and institutional affiliations. 